\title{
Corticoterapia con intención de rescate en pacientes con hepatitis autoinmune severa: ¿el trasplante hepático es evitable?
}

\author{
Corticotherapy with rescue intention in patients with severe autoimmune \\ hepatitis: is liver transplantation avoidable?
}

Corticoterapia com intenção de resgate em pacientes com hepatite autoimune grave: o transplante de fígado é evitável?

Victorio Cervera ORCID: 0000-0002-8054-6016

Médico. Especialista en Medicina

Interna y Hepatología. Past-

Fellowship Hospital Universitario

Fundación Favaloro (Buenos Aires,

Argentina). Unidad Bi-Instituciona

de Trasplante Hepático - Servicio de

Enfermedades Hepáticas HCFFAA

(Montevideo, Uruguay)

Sebastián Raffa

ORCID: 0000-0002-3466-9963

Médico. Especialista en Medicina

Interna y Hepatología. Unidad de

Hepatología y Trasplante Hepático del

Hospital de la Comunidad (Mar del

Plata, Argentina)

Valeria Descalzi

ORCID: 0000-0002-2622-063X

Médica. Especialista en Medicina

Interna y Hepatología. Unidad de

Hepatología y Trasplante Hepático

del Hospital Universitario Fundación

Favaloro (Buenos Aires, Argentina).

Marcelo Valverde

ORCID: 0000-0002-4592-0762

Médico. Especialista en Medicina Interna, Emergentología y

Hepatología del Adulto. Unidad Bi-

Institucional de Trasplante Hepático

- UDA CeNaHBP - Servicio de

Enfermedades Hepáticas HCFFAA

(Montevideo, Uruguay)
Resumen: Introducción. La hepatitis autoinmune es una enfermedad cuya presentación clínica inicial puede manifestarse de diversas formas, siendo su presentación aguda con aumento de los niveles de bilirrubina, caída de la función biosintética y necrosis masiva/submasiva o cirrosis en la histología, marcadores de severidad de la misma. La decisión de iniciar tratamiento con corticoides en este escenario resulta un desafío en la práctica clínica. Los objetivos del presente trabajo fueron evaluar el grado de respuesta al tratamiento, los predictores de respuesta, y la supervivencia global y libre de trasplante en pacientes cuyo debut clínico fue de forma aguda y severa. Resultados. Fueron incluidos 33 pacientes con bilirrubina total mayor a $2.5 \mathrm{mg} / \mathrm{dl}$, sin tratamiento previo (naive), que cumplían criterios diagnósticos según el grupo internacional de hepatitis autoinmune. El $97 \%$ eran mujeres con una mediana de edad de 52 años, el $58 \%$ se encontraba en etapa de cirrosis con un MELD promedio de 24 , y el $15 \%$ presentaba una necrosis masiva/submasiva en la muestra de biopsia hepática. En 27 casos se inició corticoterapia. El 66.7 \% evolucionó con remisión completa, 14.8 \% con remisión parcial, y en $18.5 \%$ hubo falla al tratamiento corticoideo. La colesterolemia basal y la presencia de encefalopatía hepática fueron predictores de no respuesta al tratamiento corticoideo, mientras que el MELD pre-tratamiento y la colesterolemia basal fueron las variables estadísticamente significativas asociadas a falla en el rescate del trasplante hepático. En los pacientes tratados con corticoides, la supervivencia global y libre de trasplante a 5 años en función del MELD (<25 vs $\geq 25)$ fue del $90 \%$ vs $60 \%$ respectivamente. Conclusión. Las altas tasas supervivencia logradas bajo tratamiento médico que fueron evidenciadas en esta serie reafirman la necesidad de priorizar el uso de corticoides en pacientes con hepatitis autoinmune aguda y severa.

Palabras clave: hepatitis autoinmune, corticoterapia, trasplante hepático

Abstract: Introduction. Autoimmune hepatitis can initially manifest itself in various clinical ways. Its acute presentation with increased levels of bilirubin, fall of biosynthetic function and massive/ submassive necrosis or cirrhosis in histology, is a marker of severity of it. The decision to start corticosteroid treatment in this scenario is a challenge in clinical practice. The objectives of this work were to assess the degree of response to treatment, response predictors, and overall and transplant-free survival in patients whose clinical debut was acute and severe. Results. 33 patients with total bilirubin greater than $2.5 \mathrm{mg} / \mathrm{dL}$, without prior (naive) treatment, who met diagnostic criteria according to the international autoimmune hepatitis group, were included. $97 \%$ were women with a median age of $52,58 \%$ were in the cirrhosis stage with an average MELD of 24 , and $15 \%$ had massive/submassive necrosis in the liver biopsy sample. In 27 cases treatment with cortiocosteroids was initiated, $66.7 \%$ evolved with complete remission, $14.8 \%$ with partial remission, and in $18.5 \%$ there was failure of corticosteroid treatment. Basal cholesterol and the presence of hepatic encephalopathy were predictors of non-response to corticosteroid treatment, while pre-treatment MELD and basal cholesterol were the statistically 
significant variables associated with liver transplant rescue failure. In patients treated with corticosteroids, overall and transplant-free survival at 5 years based on MELD $(<25$ vs $\geq 25)$ was $90 \%$ vs $60 \%$ respectively. Conclusion. The high survival rates achieved under medical treatment that were demonstrated in this series reaffirm the need to prioritize the use of corticosteroids in patients with acute and severe autoimmune hepatitis.

Key words: autoimmune hepatitis, corticosteroid therapy, liver transplantation

Resumo: Introdução. A hepatite autoimune é uma doença cuja apresentação clínica inicial pode se manifestar de várias formas, sendo sua apresentação aguda com níveis elevados de bilirrubina, descida na função biossintética e necrose maciça/submassiva ou cirrose em histologia, marcadores de gravidade dela. A decisão de iniciar o tratamento corticosteroide nesse cenário é um desafio na prática clínica. Os objetivos deste trabalho foram avaliar o grau de resposta ao tratamento, preditores de resposta e sobrevida geral e livre de transplantes em pacientes cuja estréia clínica foi aguda e grave. Resultados. Foram incluídos 33 pacientes com bilirrubina total superior a $2,5 \mathrm{mg} / \mathrm{dL}$, sem tratamento prévio (ingênuo), que atendiam aos critérios diagnósticos segundo o grupo internacional de hepatite autoimune. 97\% eram mulheres com idade mediana de 52 anos, $58 \%$ estavam em estágio de cirrose com um MELD médio de 24, e 15\% tinham necrose maciça/submassiva na amostra de biópsia hepática. Em 27 casos foi iniciado o tratamento médico e, em 6 casos, o transplante de fígado foi alcançado sem terapia prévia. $66,7 \%$ evoluíram com remissão completa, 14,8\% com remissão parcial e, em 18,5\%, houve falha no tratamento corticosteroide. O colesterol basal e a presença de encefalopatia hepática foram preditores de não resposta ao tratamento corticosteroide, enquanto o meld prétratamento e o colesterol basal foram as variáveis estatisticamente significativas associadas à falha de resgate do transplante de fígado. Em pacientes tratados com corticosteroides, a sobrevida geral e livre de transplante aos 5 anos com base no MELD ( $<25$ vs $\geq 25)$ foi de $90 \%$ vs $60 \%$, respectivamente. Conclusão. As altas taxas de sobrevivência alcançadas sob tratamento médico demonstrado nesta série reafirmam a necessidade de priorizar o uso de corticosteroides em pacientes com hepatite autoimune aguda e grave.

Palavras-chave: hepatite autoimune, corticoterapia, transplante de fígado 


\section{Introducción}

La hepatitis autoinmune (HAl) es una enfermedad hepática crónica de naturaleza inflamatoria, generalmente con un curso progresivo, y que se caracteriza por una serie de rasgos inmunológicos, como la presencia de autoanticuerpos circulantes y la elevación de las globulinas plasmáticas.

La dificultad en el manejo de esta enfermedad radica en la gran variedad de formas de presentación, manifestaciones clínicas, hallazgos histológicos, fenotipos inmuno-genéticos y anomalías serológicas que presenta. Se caracteriza por cursar desde formas asintomáticas o crónicas insidiosas, hasta formas agudas graves e incluso fulminantes, o presentarse en fases avanzadas, debutando como cirrosis descompensada.

La efectividad del tratamiento inmunosupresor puede variar acorde al momento en que se lo inicie y a la forma presentación, siendo aquellas formas agudas y severas, con aumento de los niveles de bilirrubina, caída de la función biosintética y necrosis masiva/submasiva o cirrosis en la histología, las de más difícil manejo ${ }^{(1)}$.

Las publicaciones acerca de pacientes con HAI severa son escasas, y la mayoría se basan en el análisis retrospectivo de pequeñas series de pacientes con resultados disímiles respecto a cuándo y cómo iniciar la terapia con corticoides, o a cuáles son las variables asociadas a una pobre respuesta a dicho tratamiento. ${ }^{(1-5)}$ Este hecho pone de manifiesto la variabilidad que existe en el pronóstico, sino también en el abordaje clínico de estos pacientes.

El presente trabajo fue dirigido a contribuir en el manejo terapéutico y en la toma de decisiones en este grupo complejo de pacientes. Los objetivos son evaluar la tasa de respuesta al tratamiento corticoideo en pacientes con HAl severa, establecer variables asociadas a dicha respuesta en este grupo de pacientes y determinar la supervivencia global y libre de trasplante hepático $(\mathrm{TH})$ en dicho escenario.

\section{Metodología}

Se realizó el análisis retrospectivo de pacientes con diagnóstico de HAl aguda evaluados consecutivamente en la Unidad de Hepatología del Hospital Universitario de la Fundación Favaloro (HUFF), entre enero del 2010 y julio del 2016.

\section{a) Criterios de inclusión}

- Edad > 15 años

- Diagnóstico de HAl según criterios del grupo internacional de HAI (GIHAI) $(6,7)$

- Forma clínica de presentación aguda

- Valor de bilirrubina $>2.5 \mathrm{mg} / \mathrm{dl}$

- Ausencia de tratamiento previo

\section{b) Variables}

Para descartar otras causas de enfermedad hepática concomitante se realizaron pruebas serológicas (virus hepatitis $A$, virus hepatitis $B$, virus hepatitis $C$, virus Epstein-Barr, citomegalovirus), estudios del metabolismo del hierro y del cobre, imagenología, e histología en aquellos pacientes biopsiados.

Los pacientes seleccionados para iniciar tratamiento médico recibieron dosis iniciales de Prednisona de 20-60 mg/ día combinado con Azatioprina 50-100 mg/día, de acuerdo con las guías y recomendaciones publicadas para el manejo de esta entidad. ${ }^{(8-10)}$

Los parámetros de laboratorio tales como bilirrubina total, ALT, globulinas, inmunoglobulina G, creatinina plasmática e INR fueron analizados al día 0 (ingreso), y a los 7, 30 y 90 días posteriores al inicio del tratamiento inmunosupresor. La albúmina plasmática, la colesterolemia, y la presencia de anticuerpos antinucleares (FAN), anticuerpos anti-mitocondriales (AMA), anticuerpos anti-músculo liso (ASMA), anticuerpos microsomales hígado y riñón (LKM1) y otros Anticuerpos (anticitosol hepático tipo 1 -LC1 y SLA/LP) fueron solicitados únicamente en la fase pre-tratamiento.

Variables clínicas como la presencia de encefalopatía hepática $(E H)$ y/o de ascitis fueron analizadas con la misma sistemática que las variables de laboratorio. 
El planteo de "sospecha clínica de cirrosis" se basó en la presencia de várices esofágicas en la endoscopía digestiva, hallazgos ecográficos compatibles (hígado disminuido de tamaño, circulación colateral, ascitis y/o esplenomegalia), y estigmas de enfermedad hepática avanzada en el examen físico.

Las biopsias hepáticas fueron obtenidas previamente al inicio del tratamiento y analizadas en conjunto por el Servicio de Anatomía Patológica y la Unidad de Hepatología del HUFF. El grado de necro-inflamación y el estadio de fibrosis fueron evaluados acorde a los criterios del GIHAl y al score de Ishak respectivamente (7).

La respuesta al tratamiento con corticoides se definió como completa, parcial o nula de acuerdo con variables clínicas, bioquímicas y parámetros de funcionalidad hepática: ${ }^{(8-10)}$

- Remisión completa: normalización de AST/ALT, bilirrubina total y gamma-globulina luego de al menos 6 semanas de tratamiento, en ausencia de peoría en el lapso de 3 semanas a 3 meses post suspensión del mismo.

- Remisión parcial: pacientes que respondieron al tratamiento, pero que requirieron dosis bajas de corticoides para el mantenimiento de la respuesta.

- Falla al tratamiento: ausencia de respuesta a dosis máximas de corticoides y azatioprina por al menos 1 mes.

\section{c) Análisis estadístico}

El análisis de las variables clínicas, bioquímicas e histológicas fue realizado por pruebas no paramétricas (Chi-cuadrado y U Mann- Whitney). Fue considerado estadísticamente significativo un valor de $\mathrm{p} \leq 0.05$.

La descripción de las variables numéricas se realizó por la mediana y su rango intercuartil (p25-p75), mientras que las variables categóricas se expresaron en porcentajes.

La supervivencia actuarial global y libre de trasplante hepático o muerte fue realizada por Kaplan-Meyer, realizándose un subanálisis según MELD $<250 \geq 25$.

Todos los datos fueron analizados con el software estadístico SPSS 17.0.

\section{Resultados}

Fueron incluidos 33 pacientes con una mediana de edad de 52 años (31.5-62), que cumplían los criterios diagnósticos modificados del GIHAl ( $\geq 6$ puntos) (7).

El $97 \%$ de los casos ( $n=32)$ fueron mujeres. El $57 \%(n=19)$ fue catalogado como cirróticos en la valoración inicial, 10 presentaron EH (30\%) y 15 ascitis (45\%).

En cuanto al perfil inmunológico, el $39.4 \%(n=13)$ presentaron ANA/FAN y ASMA positivos, $6.1 \%(n=2)$ anti-LC1 positivos, y $3 \%(n=1)$ anti-LKM1 positivos. gr/dl.

El valor de inmunoglobulina G fue 2662 (1802-3445) mg/dl y de gammaglobulina 2.6 (2-3.8)

Las variables bioquímicas al ingreso que presentaron una mediana elevada fueron la bilirrubina total $(8.8 \mathrm{mg} / \mathrm{dl})$, y el INR (1.72).

Respecto a otros parámetros, los resultados fueron los siguientes: creatininemia: 0.6 (0.5$0.8) \mathrm{mg} / \mathrm{dl}$, recuento de plaquetas: 125000 (81000-213000), natremia: 135 (132-137) mEq/L, albuminemia: 2.8 (2.3-3.2) gr/dl, y colesterolemia: 129 (79-149) mg/dl. (Tabla 1).

\begin{tabular}{|l|l|}
\hline Variable & Mediana 0 \% \\
\hline Edad (años) & $51.7(31.5-62)$ \\
\hline Cirrosis & $57 \%$ \\
\hline Encefalopatía hepática & $30 \%$ \\
\hline Ascitis & $45.5 \%$ \\
\hline LKM/LC & $3 \%-6.1 \%$ \\
\hline FAN/ASMA & $39.4 \%-39.4 \%$ \\
\hline
\end{tabular}


Tabla 1: Descripción de la cohorte en lo

\begin{tabular}{|l|l|}
\hline Nivel de lgG (mg/dl) & 2662 (1802-3442) \\
\hline Gammaglobulinas (gr/dl) & $2.6(2-3.8)$ \\
\hline Bilirrubina total (mg/dl) & $8.8(5.4-20.6)$ \\
\hline ALT & $482(135-968)$ \\
\hline INR & $1.72(1.35-2.31)$ \\
\hline Creatininemia (mg/dl) & $0.6(0.5-0.8)$ \\
\hline Plaquetas (x 103) & $125(81-213)$ \\
\hline Natremia (mEg/lt) & $135(132-137)$ \\
\hline Albuminemia (gr/dl) & $2.8(2.35-3.20)$ \\
\hline Colesterolemia (mg/dl) & $129(79-149)$ \\
\hline MELD & $24(17.5-27)$ \\
\hline
\end{tabular}

En lo que refiere a los hallazgos histológicos, en 19 pacientes se realizó biopsia hepática (57.6\%), 13 por vía transyugular (68.4\%), y 6 por vía percutánea (31.6\%). La mediana del Score de Ishak fue de $9.5(6.7-12), 21 \%(n=4)$ presentaron infiltrados plasmocitarios, $10.5 \%(n=2)$ presencia de eosinófilos, $100 \%(n=19)$ hepatitis de interfase, $21 \%(n=4)$ rosetas hepatocitarias, y $47.4 \%$ necrosis centrolobulillar (55\% severa, $11.1 \%$ moderada, 33.3\% leve). En un $26 \%(n=5)$ de los casos los hallazgos fueron compatibles con necrosis masiva/submasiva. En cuanto al grado de fibrosis, 9 pacientes presentaron estadio F4 (42.3\%), 5 F3 (26.3 \%), 1 F2 (5.2\%), 2 F1 (10.5\%) y 1 F0 (5.2\%). La valoración de la fibrosis fue dificultosa en algunos casos por la presencia de importante necrosis hepatocitaria (Gráfico 1 y 2).

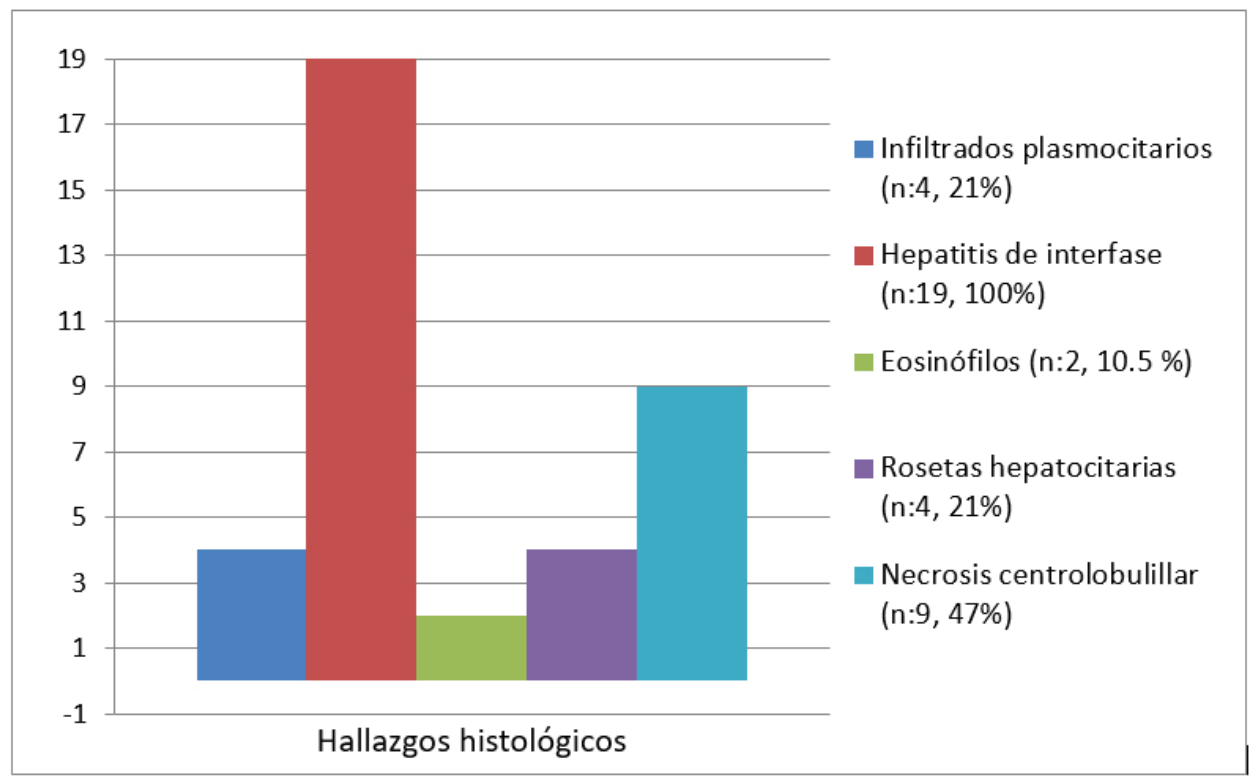


Gráfico 2: Estadio de fibrosis y necrosis masiva/submasiva en pacientes con biopsia pre-tratamiento $(n=19)$.
Figura 1: Conducta terapéutica respuesta en la población del estudio.

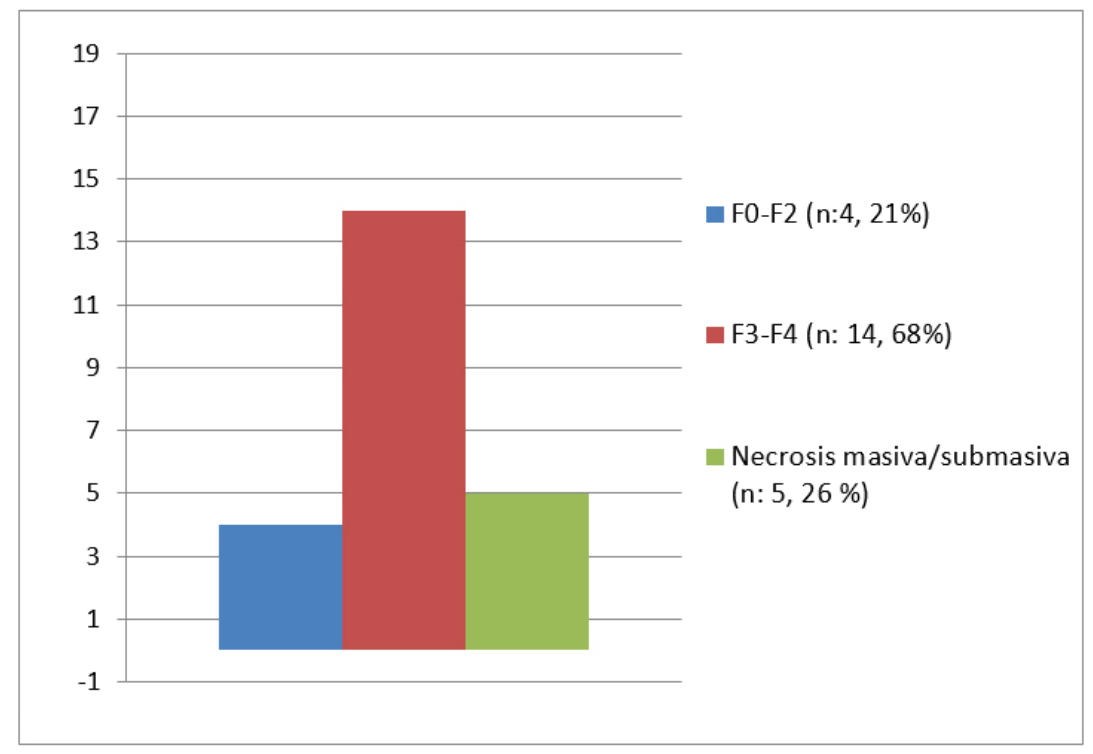

En lo que refiere al seguimiento de los pacientes, en $6(18,2 \%)$ se realizó TH de inicio, en $27(82 \%)$ se inició tratamiento con corticoides- Prednisona a 40 (20-60) mg/día, agregando posteriormente Azatioprina (50 mg/día). Siguiendo los criterios de respuesta, 18 pacientes (66.7\%) tuvieron respuesta completa, $4(14.8 \%)$ parcial, y $5(18.5 \%)$ presentaron falla al tratamiento. De los 5 pacientes no respondedores, $60 \%(3 / 5)$ requirieron $\mathrm{TH}$ por deterioro de la función hepática, y $40 \%(2 / 5)$ fallecieron por desarrollo de infecciones bacterianas intercurrentes (peritonitis bacteriana espontánea y neumonia aguda comunitaria). Figura 1.

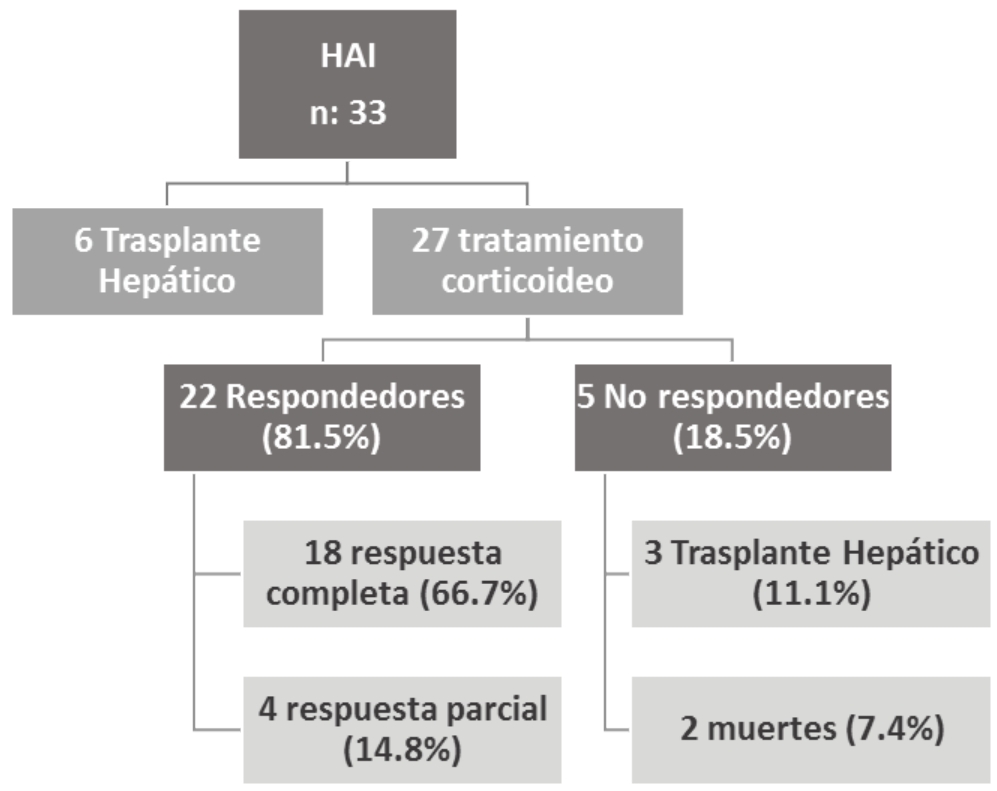

La Tabla 2 detalla la mediana (p25-p75) de las variables de laboratorio analizadas a lo largo del tratamiento en el grupo respondedor $(n=22)$ a los días $0,7,30$ y 90 respectivamente.

\begin{tabular}{|l|l|l|l|l|}
\hline Dia & $\begin{array}{l}\text { Bilirrubina total } \\
\text { mg/dl }\end{array}$ & $\begin{array}{l}\text { ALT } \\
\text { UI/I }\end{array}$ & $\begin{array}{l}\text { RIN } \\
\text { UI/I }\end{array}$ & MELD \\
\hline 0 & $8.8(5.4-20.6)$ & $482(135-968)$ & $1.72(1.35-9.68)$ & $24(17.5-27)$ \\
\hline 7 & $4.3(2.3-10.3)$ & $158(78-437)$ & $1.3(1.2-1.7)$ & $15.5(12.2-20.7)$ \\
\hline 30 & $2.25(1.52-2.95)$ & $120(56-303)$ & $1.17(1.1-1.4)$ & $11(10-14)$ \\
\hline 90 & $1.1(0.95-1.9)$ & $52(36-91)$ & $1.07(1-1.21)$ & $9(8-13)$ \\
\hline
\end{tabular}


En cuanto a los predictores asociados al "no rescate" vs "rescate" del TH, se analizaron las diferentes variables de forma dinámica $(n=33), 2$ de las cuales fueron estadísticamente significativas: el MELD pre-tratamiento (27 vs $19.5, \mathrm{p}=0,006)$ y la colesterolemia basal (83 vs $139 \mathrm{mg} / \mathrm{dl}, \mathrm{p}=0,002)$.

La ausencia de correlación entre la severidad histológica y el "no rescate" plantea que, a pesar de que presenten necrosis masiva/submasiva o cirrosis, es probable que el tratamiento corticoideo permita evitar la necesidad de TH en este grupo de pacientes.

En lo que refiere a variables asociadas a falla del tratamiento corticoideo vs respuesta al mismo, las que tuvieron significancia estadística fueron la colesterolemia basal (68 vs $139 \mathrm{mg} /$ $\mathrm{dl}, \mathrm{p}=0.004)$ y la presencia de $\mathrm{EH}$ (60 vs $18.2 \%, \mathrm{p}=0.05)$.

Respecto a la supervivencia de la cohorte analizada, luego de una mediana de 2 años de seguimiento (0.63-3.4), $22(66.7 \%)$ permanecieron vivos bajo tratamiento, sin necesidad de $\mathrm{TH}$ ("rescatados del TH"), 9 (27.3\%) fueron trasplantados (6 al inicio y 3 no respondedores). Fallecieron 3 (6\%) pacientes ( 2 no respondedores y 1 post-TH) ("no rescatados del TH") (Tablas 3 y 4$)$.

Tabla 3: Variables asociadas al "rescate" vs "no rescate" del TH con tratamiento corticoideo $(n=33)$

Tabla 4: Variables asociadas a la "respuesta" vs "no respuesta" al tratamiento corticoideo ( $n=27)$. Referencias: $\left(^{*}\right)$ Valor de la Mediana (rango intercuartil, p25-p75). $\left.{ }^{\star *}\right)$ Respondedores: Remisión Completa $(n=18)+$ Remisión Parcial $(n=4)$.

\begin{tabular}{|l|l|l|l|}
\hline & Rescatados $(\mathbf{n = 2 2})$ & No Rescatados $(\mathbf{n = 1 1 )}$ & $\mathbf{p}$ \\
\hline MELD pre-tratamiento & $19.5(15.7-26)$ & $27(25-28)$ & 0,006 \\
\hline Colesterolemia & $139(108-193)$ & $83(59-105)$ & 0,002 \\
\hline
\end{tabular}

\begin{tabular}{|l|l|l|l|}
\hline & $\begin{array}{l}\text { Respondedores } \\
(\mathbf{n}=22) * / *\end{array}$ & $\begin{array}{l}\text { No respondedores } \\
(\mathbf{n}=5) *\end{array}$ & $\mathbf{p}$ \\
\hline Bilirrubina total & $7.5(5.3-16.3)$ & $19.7(4.5-28.2)$ & 0,28 \\
\hline ALT & $320(151-816)$ & $135(52.5-1660)$ & 1 \\
\hline RIN & $1.54(1.32-2.15)$ & $1.85(1.49-2.8)$ & 0,23 \\
\hline MELD (día 0) & $19.5(15.7-26)$ & $25(22.5-26)$ & 0,16 \\
\hline Cirrosis $(n=16)$ & $81.3 \%(n=13)$ & $18.8 \%(n=3)$ & 0,97 \\
\hline Colesterolemia & $139(108-193)$ & $68(49-78)$ & 0,004 \\
\hline EH & $18.2 \%(n=4)$ & $60 \%(n=3)$ & 0,05 \\
\hline Ascitis & $36.4 \%(n=8)$ & $60 \%(n=3)$ & 0,62 \\
\hline
\end{tabular}

La supervivencia global y libre de TH o muerte a 5 años $(n=33)$ fue de $90 \%$ y $62.5 \%$ respectivamente. La supervivencia libre de eventos en función del puntaje MELD $(<25$ vs. $\geq$ 25) fue de $85 \%$ y $41 \%(p=0,001)$, mientras que la supervivencia global fue de $100 \%$ vs. $80 \%$ $(p=0,09)$ (Gráfico 3).

Gráfico 3: Supervivencia global y libre de TH o muerte a 5 años según MELD en la cohorte del estudio $(n=33)$.
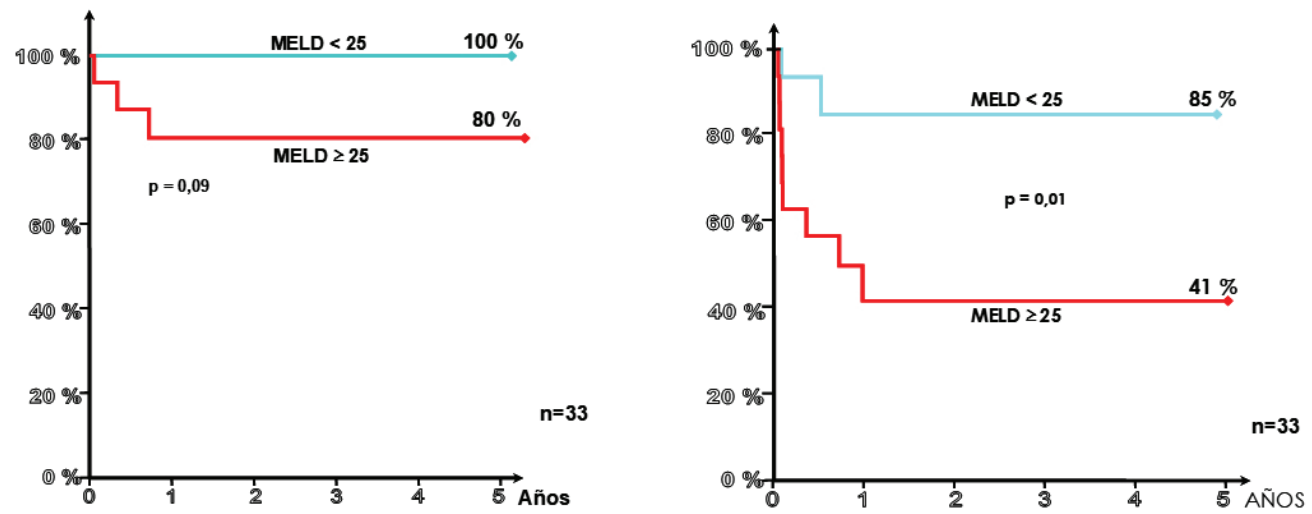

En el grupo tratado con corticoides $(n=27)$ la supervivencia global y libre de TH o muerte a 5 años fue de $92 \%$ y $77.5 \%$ respectivamente. La supervivencia libre de eventos en función del 
puntaje MELD (<25 vs. $\geq 25)$ fue de $90 \%$ y $60 \%(p=0,08)$ respectivamente, y la supervivencia global de $100 \%$ vs. $82 \%$ ( $p=0,1)$ (Gráfico 4).
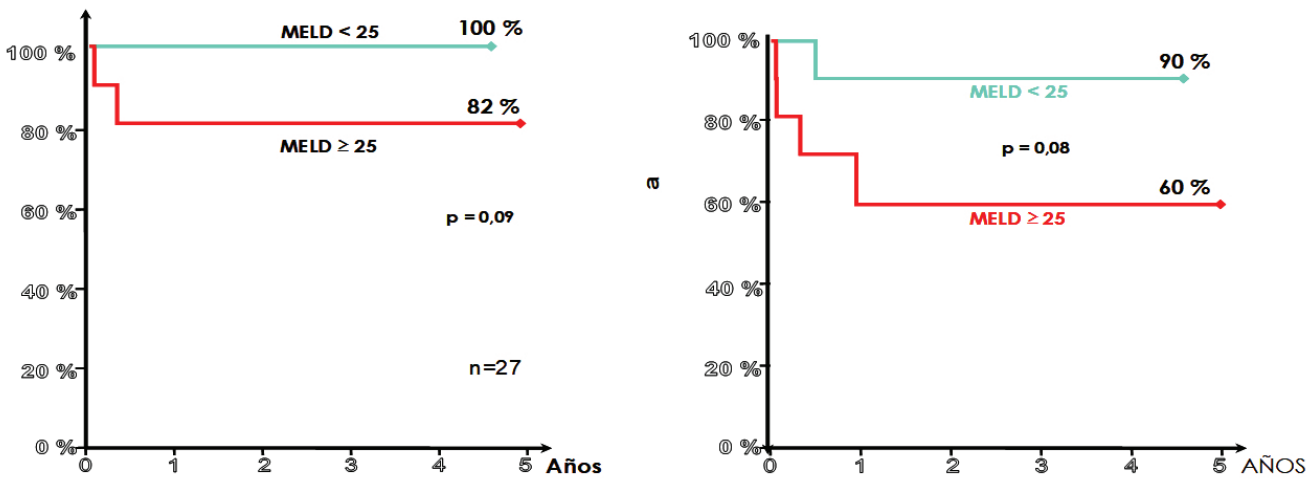

\section{Discusión}

Los pacientes con HAl severa constituyen un subgrupo difícil de tratar, y con mayores posibilidades de presentar falla al tratamiento corticoideo ${ }^{(3)}$.

En este estudio los pacientes con HAl severa presentaron un estadio histológico avanzado (necrosis masiva/submasiva, cirrosis) y un mayor deterioro de la función de síntesis hepática (ictericia, MELD elevado).

En cuanto al score de MELD, el subgrupo que mostró menor respuesta al tratamiento y supervivencia fue aquel con score $\geq 25$. Según Yeoman y col. ${ }^{(3)}$ el MELD promedio de aquellos pacientes que fallaron a la terapia con corticoides, y que presentaron peor evolución fue de $\geq 26$ (16-35). Por tanto, conocer este comportamiento es fundamental para seleccionar adecuadamente a los pacientes candidatos a tratar en un centro de referencia con posibilidades de brindar acceso a los cuidados de la hepatología crítica, incluyendo el trasplante hepático.

En el análisis de los grupos "rescatados" vs "no rescatados" del TH, las variables estadísticamente significativas asociadas con dicha respuesta fueron el score de MELD y la colesterolemia pre-tratamiento. Existen reportes que coinciden con el valor pronóstico del MELD $(\geq 26 \mathrm{p}=0,02)$ al día $0^{(3)}$. Este hallazgo abre la interrogante sobre cuál es la mejor conducta frente a este grupo de pacientes, en quienes se debe discutir si iniciar el tratamiento inmunosupresor, o avanzar hacia el trasplante hepático.

En los 27 pacientes (82\%) que recibieron corticoides se identificaron variables asociadas a falla del tratamiento. El valor de colesterol plasmático y la presencia de encefalopatía hepática pre-tratamiento fueron predictores estadísticamente significativos de falla al tratamiento. La determinación del colesterol plasmático es una prueba bioquímica que valora el grado de función de síntesis hepática, por lo cual la hipocolesterolemia puede encontrarse tanto en la hepatitis aguda grave como en la cirrosis. Su limitación clínica radica en el hecho de que no es un marcador temprano de insuficiencia hepática, por lo que podría carecer de valor en la toma de decisiones terapéuticas iniciales. En el presente estudio la hipocolesterolemia podría explicarse por la alta prevalencia de cirrosis y necrosis masiva/submasiva en este grupo de pacientes. La encefalopatía hepática se presenta como complicación tanto en la insuficiencia hepática crónica como en la aguda, pudiendo ser un evento precoz. Esto le otorga más sensibilidad como marcador de severidad y predictor precoz de falla al tratamiento. En el estudio de Villamil y col. ${ }^{(1)}$ los pacientes con EH grado 4 presentaron menor posibilidad de responder al tratamiento corticoideo. En el presente trabajo, la bilirrubina total aumentada $(7.5$ versus $19.7 \mathrm{mg} / \mathrm{dl}, \mathrm{p}=$ $0,28)$, y el INR prolongado (1.54 versus $1.85, p=0,23$ ) fueron más frecuentes en aquellos no respondedores, sin una diferencia estadísticamente significativa.

En este estudio el valor de MELD fue un predictor de respuesta al tratamiento, ya que el total de los respondedores presentaba un puntaje $<25$. En la publicación de Verma y col. (11) el total de los respondedores tuvo un score MELD inicial $\leq 28$. Del total de pacientes tratados con corticoides ${ }^{(27)}, 18(66.7 \%)$ presentaron remisión completa, y $4(14.8 \%)$ respuesta parcial, conformando el total del grupo respondedor (22) ${ }^{(8-10)}$.

El 59.1\% (13/22) de los pacientes respondedores presentaba cirrosis (por sospecha clínica o confirmación histológica) lo cual no fue determinante en la probabilidad de repuesta. Esto 
coincide con lo presentado por Yeoman y col. (3) quien no encontró hallazgos histológicos significativamente diferentes entre el grupo respondedor y no respondedor.

Realizando el análisis en función del MELD se concluyó que aquellos con un puntaje < 25 presentaron una supervivencia global del $100 \%$ y libre de TH del 90\%. En pacientes que iniciaron tratamiento con MELD $\geq 25$ la supervivencia global bajó al $82 \%$ y libre de TH a un $60 \%$. Con esta última observación se puede concluir que aún en el grupo de pacientes con HAI severa la tasa de supervivencia global es más que aceptable, lo que justificaría el intento de tratamiento con corticoides independientemente de la gravedad de la situación inicial, siempre considerando que frente a la falla de este existe la posibilidad de avanzar al $\mathrm{TH}$.

Este último concepto es de especial importancia en muchos de los países de la región, ya que la accesibilidad al TH en escenarios de falla hepática aguda está condicionada por la baja disponibilidad de órganos en los plazos adecuados.

Debido a las excelentes tasas de supervivencias halladas en este estudio, el tratamiento con corticoides es una herramienta válida en la actualidad como primera línea terapéutica independientemente de la severidad de la enfermedad.

El $18 \%$ de los pacientes no iniciaron terapia con corticoides por presentar EH grado 4 , necrosis masiva/submasiva en la biopsia hepática, o desarrollo de complicaciones infecciosas intercurrentes. Este constituye un grupo de pacientes de difícil manejo por la ausencia de estudios prospectivos multicéntricos que contribuyan en la toma de decisiones clínico-terapéuticas.

\section{Conclusiones}

La HAl de presentación aguda y severa, con aumento de la bilirrubina sérica (forma ictérica) representa un verdadero desafío médico. La decisión de iniciar el tratamiento corticoideo y el mejor momento para hacerlo es el punto de mayor discusión.

En el presente análisis los pacientes con HAI y MELD $<25$ presentaron una sobrevida a 5 años libre de eventos (TH o muerte) del $90 \%$.

A pesar de la menor sobrevida libre de TH que presentó el grupo de mayor severidad (MELD $\geq 25$ ), la tasa se mantuvo dentro de valores aceptables, cercana al $60 \%$.

Esto último reafirma la necesidad de priorizar el tratamiento con corticoides independientemente del grado de enfermedad que presenten los pacientes con HAl al momento del debut, dado que en función de los resultados de este estudio presentan una alta probabilidad de ser rescatados del $\mathrm{TH}$.

\section{Conflicto de interés}

Los autores declaran no presentar conflictos de interés para el desarrollo de este estudio.

\section{Bibliografía}

1- Villamil AG, Casciato P, Eduardo M, Bustos D, Giunta D, Ciardullo M, et al. Fulminant autoinmune hepatitis type 1: Clinical presentation, Outcome and Prognostic factors. (abstract 480) AJT 2005 (suppl 11): 278.

2- Verma S, Maheshwari A, Thuluvath P. Liver failure as initial presentation of autoimmune hepatitis: clinical characteristics, predictors of response to steroid therapy, and outcomes. Hepatology. 2009 Apr;49(4):1396-7. doi: 10.1002/hep.22894.

3- Yeoman AD, Westbrook RH, Zen Y, Maninchedda P, Portmann BC, Devlin J, et al. Early predictors of corticosteroid treatment failure in Icteric presentations of Autoimmune Hepatitis. Hepatology. 2011 Mar; 53(3):926-34. doi: 10.1002/hep.24141.

4- Miyake $\mathrm{Y}$, Iwasaki $\mathrm{Y}$, Terada R, Onishi T, Okamoto R, Sakai N, et al. Clinical characteristics of Fulminant - type Autoinmune Hepatitis: an analysis of eleven cases. Aliment PharmacolTher 2006.

5- Ichai P, Duclos-Vallée JC Ichai P, Duclos-Vallée JC, Guettier C, Hamida SB, Antonini T, Delvart V, et al. Usefulness of corticosteroids for the treatment of severe and fulminant forms of autoimmune hepatitis. Liver Transpl. 2007 Jul;13(7):996-1003. doi: 10.1002/lt.21036.

6- Yeoman AD, Westbrook RH, Al-Chalabi T, Carey I, Heaton ND, Portmann BC, et al. Diagnostic value and utility of the simplified International Autoimmune hepatitis Group (IAIHG). Hepatology. 2009 Aug;50(2):538-45. doi: 10.1002/hep.23042. 
7- Hennes EM, Zeniya M, Czaja AJ, Parés A, Dalekos GN, Krawitt EL, et al. Simplified Criteria for the Diagnosis of Autoinmune Hepatitis. Hepatology. 2008 Jul;48(1):169-76. doi: 10.1002/hep.22322.

8- Manns M, Czaja AJ, Gorham JD, Krawitt EL, Mieli-Vergani G, Vergani D, et al. AASLD Practice Guideline: Diagnosis and Management of Autoinmune Hepatitis. AASLD 2014 (Actualization 2014). Hepatology. 2010; 51(6): 1-31.

9- Potts JR, Verma S. Optimizing management in autoimmune hepatitis with liver failure at initial presentation. World J Gastroenterol. 2011 Apr 28;17(16):2070-5. doi: 10.3748/wjg.v17.i16.2070.

10- Manns MP, Lohse AW, Vergani D. Autoimmune hepatitis--Update 2015. J Hepatol. 2015 Apr;62(1 Suppl):S100-11. doi: 10.1016/j.jhep.2015.03.005

11- Verma S, Torbenson M, Thuluvath PJ. The impact of ethnicity on the natural history of autoimmune hepatitis. Hepatology. 2007 Dec;46(6):1828-35. doi: 10.1002/hep.21884.

\section{Aporte de cada autor al trabajo}

Victorio Cervera: Concepción y diseño del trabajo, recolección de datos, análisis e interpretación de los datos o resultados y redacción del manuscrito

Sebastián Raffa: Concepción y diseño del trabajo, recolección de datos, análisis e interpretación de los datos o resultados y redacción del manuscrito

Valeria Descalzi: Concepción y diseño del trabajo, recolección de datos, análisis e interpretación de los datos o resultados y redacción del manuscrito

Marcelo Valverde: Análisis e interpretación de los datos o resultados, redacción y revisión crítica del manuscrito 